http://revistaurbanismo.uchile.cl

\title{
Costos ocultos en la producción inmobiliaria
}

M. Eugenia Pallarés T.

\section{Filiación}

Académica del Departamento de Ciencias de la Construcción de la F.A.U. de la Universidad de Chile.

\section{Resumen}

La gestión de proyectos inmobiliarios corresponde a un área de negocios en que la financiación se rige por parámetros estándares, independiente de las características del proceso productivo. El ambiente de la producción de los bienes inmuebles se caracteriza por corresponder a una actividad económica que difiere de otros procesos productivos de la industria manufacturera, principalmente por la cantidad de materias primas que emplea y por los distintos valores que incorpora. La cantidad de eventos que confluyen a la ejecución de los proyectos y que no incorporan valor constituye una de las razones más importantes para el establecimiento de las tasas de descuento que se les exige a estos proyectos. Los hechos que no incorporan valor y que significan costo se cargan en el precio de venta, luego es transferido y quien finalmente lo paga es el demandante. Detectar las variables que incorporan costos, aislarlas y cuantificarlas es el objetivo principal. Consignar todos los costos efectivos de los proyectos representa el anhelo de quienes participan en este mercado. Determinar la incidencia de los costos ocultos y evitables es la forma que permitirá establecer la comparación cierta entre proyectos alternativos. Establecer la tasa de descuento real para el sector, y sus sensibilidades para las tipologías de proyectos es el resultado proyectable de este estudio

\section{Palabras Clave}

Gestión inmobiliaria, mayores costos de producción inmobiliaria.

\begin{abstract}
The subject of this work is hidden costs in property production. The principal objective is the identification, isolation and quantification of the variables which incorporate costs. The assignment of all the effective costs of projects represents the aspiration of all who participate in the market. To determine the incidence of hidden costs and avoidable is the form that will establish the true comparison between alternative projects. Establish the real discount rate for the sector, and their sensitivities to the types of projects is the projected outcome of this study.
\end{abstract}

\section{Key words}

Property management, real estate production costs, hidden costs in property production. 
http://revistaurbanismo.uchile.cl

\section{Sumario}

Introducción

Costos atribuibles al entorno

Costos atribuibles a la estructura organizacional

Costos ocultos producto de la gestión

Ajuste de tasa de descuento

Referencias

\section{Introducción}

Cuando el producto inmobiliario es transferido, lo normal es que la caracterización que de él se hace esté dada en función de lo que realmente puede usarse, medirse y verse; de allí que cuando se transfiere un bien raíz se hace la consideración de su superficie expresada en los metros cuadrados útiles, independiente de que la superficie real es mayor porque también incluye muros, tabiques, ductos, etc. Por otra parte al promocionar el producto se describen las características de materialidad que a simple vista se detectan y que corresponden a las terminaciones, en ningún escenario se habla de la estructura que lo condiciona.

La razón de esto obedece al hecho de que vender algo que no se ve resulta difícil de comprender, la tendencia del usuario es adquirir el contenido, sin cuestionar el proceso que la producción del bien involucró.

Evidentemente el precio de la transferencia no sólo involucra lo que aparentemente se entrega, sino que todo aquello que el proceso usó. En el proceso de producción, ocurre una situación similar, donde la segmentación de los costos se da bajo diferentes criterios, siendo quizás el más común aquel que los separa en función de lo que materialmente se incorpora y que se conoce como costo directo, y aquello que se requiere pero que no es parte del proceso constructivo y que se conoce como costo indirecto.

La internalización de este matiz tiene etapas, y dependiendo del mercado, es posible que se asuma que, por ejemplo, el costo indirecto del proyecto de arquitectura constituye un valor vendible, situación que en los segmentos más altos es frecuente, pero que en los más bajos no se entiende y mucho menos valora, de allí que independiente de su presencia y del costo que significa para el producto - el cual porcentualmente es similar en ambos nichos - la sensibilidad del comprador se resiste a pagarlo, luego se oculta para no desvirtuar la transacción. Ejemplos de esta situación se muestran en la publicidad que, cuando se orienta a productos para el segmento socio económico $A B$, destaca al proyectista, y cuando lo hace para los tramos $C 3$ y $D$, no lo menciona, evidentemente en un caso se apela al valor que el diseñador aporta a la producción y que se transmite como precio, en el otro escenario sólo es un costo.

Esto que pareciera una cuestión común a muchos bienes de consumo masivo, para los productores puede llegar a constituirse en una barrera en el acceso a financiamiento, ya que la posibilidad de señalar en cada proceso en forma pormenorizada cada una de 
las fuentes de costos suele resultar extenso y más aún difícil de entender, sobre todo porque las incidencias individuales resultan de todas las magnitudes.

Más complejo aún es estimar y declarar costos que ocurren en el proceso y que, o son propios del ejecutante, o dicen relación con el entorno, en ambas situaciones y desde la perspectiva del productor, la actitud es que ante la posibilidad de ocurrencia, situación evidentemente de riesgo, la manera de resolverla es cargándola en la tasa de descuento, y es allí precisamente donde la competencia por la financiación hace que el sector pierda posición, porque es sabido que la expresión de riesgo de los proyectos se incorpora en la tasa de descuento que se les exige, luego a mayor tasa de descuento mayor riesgo, por lo tanto conseguir financiamiento resulta más difícil ya que los inversionistas privados requieren certeza y en tiempos de crisis son fundamentalmente adversos al riesgo, además que los inversionistas institucionales responden a gran número de asociados por lo que independiente de su percepción ante el riesgo, desde el punto de vista normativo requieren de certidumbre.

Si en la competencia por la financiación, uno de los matices es la alta tasa de descuento y si sabemos positivamente que una de sus causales es la presencia de costos ocultos, una forma de competir en igualdad de condiciones con otras áreas de la producción es logrando identificar y cuantificar las fuentes de costos ocultos de manera que al aislarlas se puede fijar la verdadera tasa de descuento, que indudablemente resultará menor y por consiguiente más competitiva.

\section{Costos atribuibles al entorno}

Si analizamos el problema desde la perspectiva del entorno podemos identificar como fuentes de costos la interacción de la producción inmobiliaria con el aparato regulador público representado por las Direcciones de Obras Municipales, las Secretarías Regionales Ministeriales del Ministerio de Vivienda y Urbanismo y la División de Desarrollo Urbano del mismo Ministerio. La primera instancia indispensable para el inicio del proceso constructivo y para su posterior recepción, las otras con participación variable dependiendo de los casos y situaciones que en el proceso se dieren.

La secuencia de acciones indica que, previo a cualquier ejecución, es necesario contar con el permiso de edificación y este, que está normado en cuanto a los antecedentes que requiere para su aprobación, también está normado en el plazo en que se debe fallar. Sin embargo, y por múltiples razones derivadas de los recursos con que los Municipios cuentan, de la capacitación de sus funcionarios, de los eventos que se generan en la interacción con otras instancias de poder 0 de interés, de las expectativas de la comunidad, de las coyunturas -que hacen que en determinadas ocasiones se produzcan situaciones que desvían la atención a problemas puntuales y explosivos como ha sido en el último tiempo la dedicación a la Ley 15.583 que permite regularizar edificaciones bajo ciertas garantías fiscales-, y también, en alguna medida, por la constante y variable interpretación y discrecionalidad en la aplicación de las normativas generadas en cada uno de los organismos públicos, los plazos no se cumplen, situación que conlleva a atrasos en los inicios, los que se traducen en mayor tiempo y, por lo tanto, mayor costo ya que la inversión -que a lo menos en terreno y proyectos ya está realizada- demorará más tiempo en ser recuperada y para el 
http://revistaurbanismo.uchile.cl

inversionista significará pérdida de valor de su capital producto de tenerlo inmovilizado.

Esta circunstancia genera que en una primera instancia aumente el período de vida del proyecto, lo cual también incurrirá en mayor gasto producto de la espera a la puesta en marcha de la producción, indudablemente este mayor costo se transmite y termina pagándolo el que adquiere el bien.

Las estimaciones de atraso en el inicio de proyectos como consecuencia del otorgamiento del permiso de edificación varían en tiempo dependiendo del Municipio en que se tramite y de las características de los proyectos, pero lo prácticamente estándar dado por el seguimiento de casos en distintas localidades y períodos de tiempo fluctúa entre uno y ocho meses y que independiente de los causas que los justifican, en rigor afecta al precio final del producto.

La contribución de este ítem a la tasa de descuento es posible de medir al considerar el efecto que cada mes adicional provoca en el período de vida del proyecto, teniendo en cuenta que se produce al inicio del proyecto, ocasión en que las unidades monetarias son más incidentes en el efecto de los resultados de término.

La estimación estadística nos entrega como valor medio en los extremos de uno y ocho meses adicionales un diferencial de 0,8049 y 5,6379 repercutibles en la tasa de descuento, efectos muy fuertes independiente del tipo de producto y que llevados a precio hacen que los bienes se transen a valores incrementados por un concepto que no es propio del sistema productivo y menos aún de las intenciones del área de negocios, es absolutamente consecuencia del medio y su ineficacia.

Cabe entonces preguntarse como promotor inmobiliario, asumiendo que es este el escenario, ¿cuál debiera ser el norte? Evidentemente demostrar la falencia pero, en el intertanto, asumir el peor de los escenarios, e independiente de la localización y de la experiencia en la tramitación, resulta más seguro asumir el peor; ahora, si no se da, el excedente permite aumentar las posibilidades de éxito en la comercialización con estímulos factibles de asumir para enfrentar a la competencia, o enfrentar con recursos los períodos de error entre lo planificado y lo ocurrido en el proceso de venta, o en tiempos de crisis mejorar la oferta a través de descuentos y rebajas o diseñar modelos alternativos a la venta que permiten alargar el período de vida del proyecto sin perjudicar el retorno esperado. Evidentemente en tiempos de plena competencia obtener excelentes rentabilidades capaces de atraer a múltiples inversionistas, claro que desde la perspectiva del productor; pero para el demandante la situación genera un pago superior al costo y su adecuada retribución.

Pero, el agente público también interactúa al final del proceso productivo con ocasión de la recepción de las obras, instancia indispensable para los efectos de formalización y legalización de las ventas y transferencias, circunstancia que permite percibir los ingresos por concepto de créditos y subsidios estatales si los hubiere, ambos factores determinantes debido a que la mayoría de las operaciones se realizan a través de algunos de los sistemas de crédito que el sistema financiero ofrece, y es en esta 
http://revistaurbanismo.uchile.cl

circunstancia cuando los atrasos y las incompatibilidades entre lo planificado y lo que en el ejercicio ocurre afecta con mayor fuerza al flujo de ingresos del proyecto.

El rezago en el pago de los créditos hipotecarios es de alrededor de treinta días y en caso de los subsidios tres meses, estas son cifras conocidas y aceptadas, la dificultad se produce con las fechas aleatorias en el atraso de la recepción y que varían en los distintos Municipios y en la mayoría de los casos obedecen a solicitud de nuevos requisitos que técnicamente son posibles de cumplir, pero sin embargo involucran tiempo y, por lo general, son aceptados independiente de la pertinencia, el problema más grave que se pretende evitar son las pérdidas financieras.

Esta suerte de conformidad con las medidas obedece a que las instancias de reclamaciones son, por lo general, de mediano y largo plazo que, si bien resuelven la discusión técnica, no consideran la pérdida financiera producida, debido a lo extemporáneo del resultado.

Como es evidente, en la medida que las dificultades tengan consecuencia en las diferentes épocas del proyecto, el efecto sobre la inversión suele ser distinto, en los comienzos de la operación la inversión realizada corresponde por lo general a terreno y proyectos, ambos financiados con recursos propios - por lo tanto más caros - pero cuando ocurre al término de la etapa de construcción - ocasión de la recepción - la financiación también ha sido con partes de créditos que, mientras no se amorticen, generan intereses que, a pesar de ser calculados como costos, el sobredimensionamiento puede convertirlos en una carga excesiva.

Fuerte es también la cuantificación de la inversión realizada a cada fecha, para los efectos de solicitud de permiso municipal, la inversión se acerca como media al $20 \%$ de la inversión total, con ocasión de la recepción se está casi en el $85 \%$ de la inversión total, luego independiente de la estimación porcentual del efecto, la gravedad es mayor porque se produce en un instante en que no existe opción de retorno, y menos aún de abandono. La magnitud del efecto arroja una media de $7.46 \%$ para el primer mes, cifra que crece exponencialmente.

Un efecto de carácter secundario, pero no menos importante, es el de los compromisos asumidos en la venta en verde, instancia en que se ha establecido fecha de entrega y se ha estructurado el pago del pie en cuotas que recogen ese dato, lo normal es establecer penalizaciones a los incumplimientos, luego el atraso en la recepción podría afectar también este flujo, la incidencia en estos casos es muy baja y bordea $0,2 \%$ por cada tres meses de atraso, para venta en verde de hasta un $50 \%$ distribuido de acuerdo a la función normal. Ahora un efecto no cuantificado pero recientemente descubierto es el producto del incumplimiento en la fecha de entrega afecta a proyectos futuros de la compañía, haciendo que la negociación de los contratos de venta anticipada para nuevos proyectos se les exija mayores garantías producto de la experiencia anterior.

A la inversa cuando estos trámites suelen ser efectivos y acogidos a plazo, la entrega anticipada es una ventaja que la compañía resalta y que le permite potenciar acciones futuras. 


\section{Costos atribuibles a la estructura organizacional}

Pareciera ser que los costos atribuibles a la organización de la empresa son lo que se entiende por gastos generales y que corresponden fundamentalmente a los servicios que requiere el proyecto y que se expresan en un porcentaje de los costos directos e indirectos, este factor suele tener gran variabilidad dependiendo del tipo de empresa y su estado de afiatamiento en el tiempo y en la producción, es normal encontrarse con el hecho de que empresas que llevan bastante tiempo elaborando un tipo de productos en una determinada localización el precio de sus servicios sea constante y ajustado a valor entre el $2 \%$ y el $3 \%$ del presupuesto, cifra que llega hasta el $7 \%$ cuando se trata de empresas que recién se incorporan a un determinado mercado.

Las razones de la variabilidad suelen ser atendibles y representan el costo de lo desconocido, ahora la cuestión que no parece ética es que al comparar dos empresas en el mismo nicho y con el sesgo de la experiencia entre ellas, ambas terminan vendiendo a precio donde han transmitido a los usuarios su propia situación múltiples ejemplos encontramos en el área de renovación urbana de la Comuna de Santiago, donde productos iguales el matiz de $1 \%$ y hasta $5 \%$ más baratos representa el conocimiento que se tiene del negocio y de su estructura de costos. Resulta especial que el usuario pague la incorporación del nuevo actor al mercado, lo ético es que el precio este en el rango del más eficiente y que los que no alcanzan ese precio asuman el costo de su incorporación al mercado sin cargárselo a los demandantes.

En condiciones de competencia perfecta el mínimo diferencial en el precio hace que los productores reordenen su estructura, de lo contrario corren el riesgo de quedar fuera del mercado; ahora, cuando la demanda está deprimida y la oferta se mantiene, aquellos cuya estructura organizacional es más costosa y por lo tanto implica mayor gasto, la única forma que tienen de salir es castigando sus expectativas de rentabilidad, de lo contrario son absorbidos por otros actores.

Pareciera entonces que el mercado de la producción inmobiliaria privilegia a los actores consolidados y desincentiva a los nuevos, cuestión que también es lugar común de otras áreas de la producción, pero que al igual que en ellas, es posible de romper en la medida en que la incorporación se produzca con creatividad, con estructura dimensionada para el proyecto, con proyección de largo plazo y fundamentalmente con claridad de los matices del sector, esto es la clave para romper las barreras de entrada que el nicho aporta y que obligadamente el recién incorporado debe conocer.

Sabido es que frecuentemente las empresas que se encuentran con capacidad ociosa tienden a disfrazar la incorporación de ese lucro cesante a los proyectos en marcha, evidentemente encareciéndolos. Por otra parte, y como en períodos de crisis la competencia suele ser mucho más dura, las empresas que están recién partiendo con estructuras bastante más simples y pequeñas y que no tienen el trauma de los despidos desde el punto de vista financiero, la posibilidad de adaptación y competitividad las hace más eficientes y les permite romper la barrera de entrada con precio, cuestión que consiguen nada más que porque la competencia aumentó su tasa 
http://revistaurbanismo.uchile.cl

al estimar posibles mayores costos por la reserva de justificación de mayores gastos generales.

En el escenario de demandas deprimidas y dificultad de acceso a financiamiento, poder establecer fehacientemente la tasa de descuento del proyecto evitando los sobredimensionamiento suele ser una oportunidad y una especial situación que permite la incorporación de empresas a mercados que en escenarios normales resultaría muy difícil.

La magnitud de la variable costo oculto producto de la organización es tan diversa como el tipo de empresa que analicemos y en la temporalidad del análisis; sin embargo la vía más clara de aproximación se da en la declaración de las partidas de imprevistos que habitualmente responden a cifras estudiadas por la empresa y que de alguna manera reflejan sus niveles de competitividad.

Del análisis de varias empresas agrupadas por tamaño, experiencia y "marca", entendiendo por tal el estilo de productos que entregan, se pudo deducir que la injerencia de este factor representa en la menor rentabilidad entre el 2,5\% y el 35,7\% - este último valor resultó del análisis de un negocio en que la rentabilidad esperada es muy alta (356\%) producto de la forma de financiamiento y de las condiciones de venta en el instante de la evaluación, en cualquier caso constituye una excepción en los casos estudiados, en ellos la media se encuentra alrededor del 3,5 como factor de incidencia en la tasa que se exige a los proyectos.

Otra fuente de costo oculto y asociado a la organización es el que hemos llamado de la planificación, y que dice relación con el tiempo, los recursos y la oportunidad con que se estudian las decisiones de inversión inmobiliaria; existe una máxima que estima necesario el estudio de la mayor cantidad de variables que incorporan precio en el producto como una manera de aislar las sorpresas de la improvisación, esto que evidentemente es beneficioso tiene como dificultad, asociada el tiempo que toma cada estudio, que puede transformarse en una carga financiera muy alta, o lo que es peor, puede que signifique quedar fuera del negocio por una cuestión de oportunidad.

Lo normal es que al evaluar los proyectos inmobiliarios se considere como fuentes de costos aquellas que se inician con la adquisición del suelo y de allí en adelante se calendarice cada uno de los eventos de la producción, dejando afuera el estudio y todas las tramitaciones que llevaron a decidirse por ese suelo, la razón de no colocarlo es, en la mayoría de los casos, por estimarlo bajo, además, porque su incorporación en alguna medida representa la estructura de análisis de la empresa, tema que en algunos escenarios puede llegar a constituirse en su principal estrategia.

La cuantificación de la variable se encuentra entre el 0,5 y 3 de la tasa y se estima que debe tener un incidencia de alrededor del 1 , lo que permite traducirla directamente en costo que aporta valor al proyecto, porque el eliminarla tendría repercusión en otros factores, como por ejemplo el de imprevistos, pos venta, o el que es peor mayor período de ventas.

\section{Costos ocultos producto de la gestión}


http://revistaurbanismo.uchile.cl

En el proceso de llevar a término un proyecto inmobiliario hay actores que intervienen en la interacción con el medio y en la ejecución del producto, en ambos ambientes hay sensibilidades que pueden incorporar precio que justifique responder adecuadamente a los compromisos de plazos de

entrega y de calidad. Los que dicen relación con pagos no declarables que permitan avanzar en los procesos, en nuestro medio no son conocidos como media estadística, y es más, son muy poco mencionados y menos aún denunciados, lo que nos hace suponer que constituyendo una amenaza aún no representan un factor a estimar, cuestión que sí ocurre en otras localizaciones.

En el ámbito de la "gestión" la variabilidad de información que entregan las distintas empresas estudiadas es enorme y tiende al concepto de "caja negra", donde aparentemente representa una serie de cuestiones que pueden pasar y que no son parte del ítem "imprevistos" que se asume ligado a la construcción propiamente tal, además de que los eventos que asume son controlados directamente por el promotor y que representan la responsabilidad del primer propietario, responsable legalmente de la producción correcta del bien. Es frecuente encontrarse con que el ítem "pos venta" es parte del área gestión, que también lo son los eventuales conflictos que existiesen con posterioridad a la transferencia, además de una serie de detalles menores como la implementación de la administración en la primera etapa en que aún no han sido transferidas todas las unidades.

En la práctica, es tremendamente variable en su formulación y representa alrededor de 3,0 de la tasa de los cuales a lo menos 1,5 son evitables si durante el proceso de ejecución se mantienen los controles que establece la normativa.

\section{Ajuste de tasa de descuento}

La síntesis de los datos recogidos permite señalar las medias descritas por sector, el efecto promedio sobre la tasa hace que los costos ocultos estudiados impongan como media entre un 7,5 y 9,5 puntos de incidencia sobre la tasa, cifra que permite comprender las exigencias de tasa con alrededor de 10 puntos sobre otros negocios. Ahora, y dado que el medio está acostumbrado a esta forma de enfrentar la producción, es un tanto complejo insistir que la producción inmobiliaria tiene un costo mayor que en el que en el papel aparece, y con la teoría exclusiva del riesgo - que no desconocemos - la posibilidad de competir por los recursos se hace cada vez más difícil.

Transparentar la información también puede producir una variable más de competencia, y lo que es peor, competencia en que el factor de diferenciación es la inoperancia externa o interna, cuestión a la que evidentemente los actores del mercado no están dispuestos a enfrentar, a no ser que sean líderes $y$, por consiguiente, el efecto no les produzca daño.

Noviembre de 2000.

\section{Referencias}


http://revistaurbanismo.uchile.cl

SAPAG CHAIN, Nassir y SAPAG CHAIN, Reinaldo, en Preparación y Evaluación de Proyectos, Editorial McGraw-Hill Interamericana SA - 1998.

JANSSON, Axel, en Evaluación Privada de Proyectos, Editorial Facultad de Ciencias Económicas y Administrativas, Universidad de Chile (en proceso de publicación).

DONOSO Y OLIVA, en Alianzas estratégicas para la comercialización de un nuevo producto inmobiliario: Colegios de la reforma, Tesis para optar al grado de Master en Dirección de Empresas Constructoras e Inmobiliarias - MDI - septiembre 1999, Escuela de Posgrado, F.A.U. Universidad de Chile - Universidad Politécnica de Madrid.

PRADO, Cristóbal, en El permiso de construcción y el costo de la burocracia municipal, Tesis para optar al grado de Master en Dirección de Empresas Constructoras e Inmobiliarias - MDI - septiembre 1999, Escuela de Posgrado, F.A.U. Universidad de Chile - Universidad Politécnica de Madrid.

PALLARÉS, M. Eugenia en «Opciones de inversión inmobiliaria en el mercado chileno Políticas Públicas y Financiación» - Ponencia para el «IV Congreso Internacional de Egresados MDI» - Octubre 1999, Madrid, España.

PALLARÉS, M. Eugenia, VALENZUELA, Marcelo, "Reflexiones - Vivienda Industrializada: Una hipótesis de desarrollo", en revista "De Arquitectura" No 3, F.A.U. Universidad de Chile, octubre 1992.

CARRASCO POBLETE, Julián, en Tributación de la actividad de la construcción, Documento de Trabajo, Cámara Chilena de la Construcción - 1999.

CÁMARA CHILENA DE LA CONSTRUCCIÓN, Documento de Trabajo, Edificación Habitacional en Chile entre 1941 y 1999.

Nota: Este escrito fue desarrollado en el contexto del proyecto de investigación "Cuantificación e Incidencia de variables de costos que no incorporan valor en el producto inmobiliario." Financiado por Concurso de Investigación Facultad de Arquitectura y Urbanismo, y realizado entre los meses de marzo del 2000 y marzo del 2001. El investigador responsable es M. Eugenia Pallarés Torres y los Co - investigadores son Mario Torres Jofré y Marcelo Valenzuela Vargas. 